\title{
Expression of ASAP1 and FAK in gastric cancer and its clinicopathological significance
}

\author{
QIONG LUO ${ }^{1}$, SUYUN ZHANG $^{1 *}$, DONGHUAN ZHANG $^{1 *}$, FANG YUAN $^{2}$, XIANGQI CHEN ${ }^{2}$ and SHENG YANG $^{1}$ \\ Departments of ${ }^{1}$ Oncology Medicine and ${ }^{2}$ Respiratory Medicine, Fujian Medical University Union Hospital, \\ Fuzhou, Fujian 350001, P.R. China
}

Received September 7, 2019; Accepted March 6, 2020

DOI: $10.3892 / \mathrm{ol} .2020 .11612$

\begin{abstract}
The present study aimed to analyze the expression levels of adenosine diphosphate ribosylation factor guanylate kinase 1 (ASAP1) and focal adhesion kinase (FAK) in gastric cancer (GC) tissues in order to explore their association with clinicopathological features and prognosis. A total of 32 patients with GC were enrolled in the present study. All patients had complete clinical follow-up data and paraffin-embedded normal gastric mucosal tissues. The expression levels of ASAP1 and FAK in these tissues were measured by immunohistochemistry. The associations of ASAP1 and FAK expression with clinicopathological factors and the survival of patients with GC were subsequently analyzed. The expression levels of ASAP1 (59.4\%) and FAK $(68.8 \%)$ in GC tissues were significantly higher than those in normal gastric mucosal tissues $(28.1$ and $40.6 \%, \mathrm{P}<0.05)$. The expression levels of ASAP1 and FAK were associated with depth of invasion, lymph node metastasis and pathological stage $(\mathrm{P}<0.05)$. ASAP1 expression was positively associated with FAK expression $(\mathrm{P}<0.001)$. In addition, ASAP1 and FAK expression levels were negatively associated with disease-free survival time and overall survival time $(\mathrm{P}<0.05)$. The 5 -year overall survival rate was significantly higher in patients with negative ASAP1 or FAK expression compared with that in patients with positive ASAP1 or FAK expression $(\mathrm{P}<0.05)$. In conclusion, ASAP1 and FAK were highly expressed in human GC tissues and may serve a synergistic role in promoting
\end{abstract}

Correspondence to: Professor Sheng Yang, Department of Oncology Medicine, Fujian Medical University Union Hospital, 29 Xinquan Road, Fuzhou, Fujian 350001, P.R. China

E-mail: dryangxh@126.com

Professor Xiangqi Chen, Department of Respiratory Medicine, Fujian Medical University Union Hospital, 29 Xinquan Road, Fuzhou, Fujian 350001, P.R. China

E-mail: drchxqtg@126.com

*Contributed equally

Key words: adenosine diphosphate ribosylation factor guanylate kinase 1 , focal adhesion kinase, gastric cancer, prognosis tumorigenesis, progression, invasion and metastasis in patients with GC. ASAP1 and FAK expression in GC were associated with patient's survival. Therefore, ASAP1 and FAK may represent novel molecular markers for the pathophysiology and prognosis of GC.

\section{Introduction}

Gastric cancer (GC) is the fifth most common malignancy in the world with 951,600 new cases (6.8\% of total) and 723,100 deaths $(8.8 \%$ of total) in $2012(1,2)$. Due to the non-specific symptoms of early-stage GC, and patients with advanced GC exhibit poor prognosis. Therefore, it is necessary to identify novel specific and sensitive biomarkers for GC that can assist in early diagnosis and improve its prognosis $(3,4)$.

Adenosine diphosphate ribosylation factor guanylate kinase 1 (ASAP1) influences tumor cell migration and invasion, and therefore promotes the metastasis of tumor cells in the body. ASAP1 expression is significantly upregulated in various tumors and is closely associated with the malignant biological behavior and prognosis of these tumors (5-9). However, to the best of our knowledge, ASAP1 expression in GC and its association with GC prognosis have not been previously reported. Focal adhesion kinase (FAK) is an important non-receptor tyrosine kinase that influences cell proliferation, adhesion, invasion and migration, and is associated with tumor growth, anti-apoptotic mechanisms and tumor recurrence (10-12). Previous studies have demonstrated that FAK expression is upregulated in thyroid, esophageal, breast, gastric and intestinal cancer, and its expression is associated with a poor tumor prognosis $(13,14)$. The present study used immunohistochemistry to detect the expression levels of ASAP1 and FAK in GC tissues, and analyzed their associations with multiple clinicopathological factors and with GC prognosis.

\section{Materials and methods}

Patients and tissue samples. All clinical GC tissue samples were isolated from 32 patients who had received a subtotal gastrectomy or radical total gastrectomy between December 2011 and February 2012 at the Union Hospital of Fujian Medical University (Fuzhou, China). The patients enrolled in the present study had not received radiotherapy or chemotherapy 
before surgery. In addition, all patients had complete follow-up data and available paraffin-embedded normal gastric mucosal tissues. The follow-up rate was $100 \%$.

The clinical staging was evaluated based on the International Union Against Cancer (UICC) Tumor-NodeMetastasis (TNM) Classification of Malignant Tumors (15). Ethical approval for the present study was obtained from the Union Hospital of Fujian Medical University Ethics Committee. All patients or their guardians provided written informed consent.

Clinical information of the 32 patients is presented in Table I. There were 23 males and 9 females. The median age of the patients was 63.9 years (range, 40-87 years; patients $\geq 60$ years, $n=23$; patients $<60$ years, $n=9)$. Pathological findings indicated that $53.1 \%(n=17)$ of the tumors were classified as being moderately or highly differentiated, and $46.9 \%$ $(n=15)$ as undifferentiated or poorly differentiated. A total of 22 patients $(68.8 \%)$ presented with lymph node metastasis (N1-4). The depth of invasion was pT1+2 in 15 patients $(46.9 \%)$ and pT3+4 in 17 patients (53.1\%). A total of 14 patients $(43.8 \%)$ were allocated to TNM stage I+II and 18 patients $(56.3 \%)$ to stage III+IV.

Disease-free survival (DFS) time was defined as the time from surgery to recurrence or mortality from any cause during the 5 years of follow-up. Overall survival (OS) time was defined as the time between initial surgery and the day of the last follow-up or mortality from any cause during the 5 years of follow-up. The mean 5-year DFS time was 77.37 \pm 35.08 months and the mean 5-year OS time was $77.57 \pm 34.02$ months.

Immunohistochemistry. Serial 3- $\mu \mathrm{m}$ thick sections were cut from tissue samples, mounted onto glass slides, dewaxed in xylene and rehydrated in different concentrations $(100,95$, 90,85 and $70 \%$ ) of ethanol. The sections were autoclaved in in citrate buffer ( $\mathrm{pH} 6.0$ ) at $121^{\circ} \mathrm{C}$ for $10 \mathrm{~min}$ for antigen retrieval. Samples were blocked with $5 \%$ normal goat serum (cat. no. KL-D1418; Kalang Biologicals) at $37^{\circ} \mathrm{C}$ for $30 \mathrm{~min}$ and then incubated with primary anti-ASAP1 (1:250; cat. no. 125729; Abcam) and anti-FAK (1:250; cat. no. 40794; Abcam) overnight at $4^{\circ} \mathrm{C}$. Subsequently, samples were rinsed in PBS three times and incubated with a horseradish peroxidase-labeled secondary antibody $(1: 1,000$; cat. no. K4003; Dako; Agilent Technologies, Inc.) at $37^{\circ} \mathrm{C}$ for $30 \mathrm{~min}$. The slides were stained with 3,3'-diaminobenzidine (Dako; Agilent Technologies, Inc.) at room temperature for 2 min. Slides were washed in PBS three times. For color development: DAB color kit (cat. no. C520017; Sangon Biotech), reaction solution $(10 \mathrm{ml})$ reagents $\mathrm{A}(200 \mu \mathrm{l})$ and reagents $\mathrm{C}(20 \mu \mathrm{l})$ were used to form the DAB color development solution. After rinsing with $50 \mu \mathrm{l}$ extra water, the color development solution was added to each section in a dark place at $37^{\circ} \mathrm{C}$ for $\sim 10 \mathrm{~min}$. The sections were rinsed with purified water three times to terminate the color development. Hematoxylin (cat. no. E607318; Sangon Biotech) staining was performed at $37^{\circ} \mathrm{C}$ for $5 \mathrm{~min}$ and then counterstained with eosin (cat. no. E607318; Sangon Biotech) at $37^{\circ} \mathrm{C}$ for $30 \mathrm{sec}$. Slides were then dehydrated in $95 \%$ ethanol twice for $5 \mathrm{~min}$ each, and washed in xylene twice for $5 \mathrm{~min}$ each. Finally, sections were sealed using neutral balsam and left to dry naturally.
Table I. Clinicopathological characteristics of patients with gastric cancer.

\begin{tabular}{lrc}
\hline Characteristic & $\mathrm{n}$ & $\%$ \\
\hline Age, years & & \\
$\quad<60$ & 93 & 28.13 \\
$\geq 60$ & & 71.88 \\
Sex & 9 & \\
Female & 23 & 28.13 \\
Male & & 71.88 \\
Invasion depth & 14 & \\
pT1+2 & 18 & 43.75 \\
pT3+4 & & 56.25 \\
Lymph node metastasis & 10 & 31.25 \\
No & 22 & 68.75 \\
Yes & & \\
TNM stage & 15 & 46.88 \\
I+II & 17 & 53.12 \\
III+IV & & 53.12 \\
Degree of differentiation & 17 & 46.88 \\
Moderate/high & 15 & \\
Undifferentiated/low & & \\
\hline
\end{tabular}

pT1+2, tumor invades the submucosa or the muscularis propria; pT3+4, tumor invades the subserosa, non-peritonealised pericolic or perirectal tissues, or tumor directly invades other organs or structures and/or perforates the visceral peritoneum.

Immunohistochemistry scoring. Immunohistochemistry staining of ASAP1 and FAK was performed and scored by two experienced pathologists using a light microscope at x 200 magnification, according to a previous study published by Hou et al (5). The staining intensity was graded as follows: 0 , unstained; 1, low signal (light yellow); 2, moderate signal (yellow brown); and 3, strong signal (brown). In addition, the score associated with the percentage of positive cells was assigned as follows: $0,<5 \%$ positive cells; $1,5-10 \%$ positive cells; $2,11-50 \%$ positive cells; $3,51-80 \%$ positive cells; and 4 , $>80 \%$ positive cells. The final score was calculated by multiplying the scores associated with the percentage of positive cells by the score associated with the intensity. The scores were divided into the negative expression (final score, $0-4$ ) and positive expression (final score, 6-12) groups.

Statistical analysis. All data were analyzed using SPSS v11.5 (SPSS Inc.). Quantitative data are presented as the mean \pm SD, and qualitative data are presented as the rate or ratio. The survival time is presented as the median and quartiles. The $\chi^{2}$ test or Fisher's exact test were used to analyze the differences among groups. The $\kappa$ value was used to investigate the association between variables. Kaplan-Meier curves were used to evaluate the 5-year DFS rate or 5-year OS rate, and the log-rank test was used to analyze differences in survival rates. $\mathrm{P}<0.05$ was considered to indicate a statistically significant difference. 
A

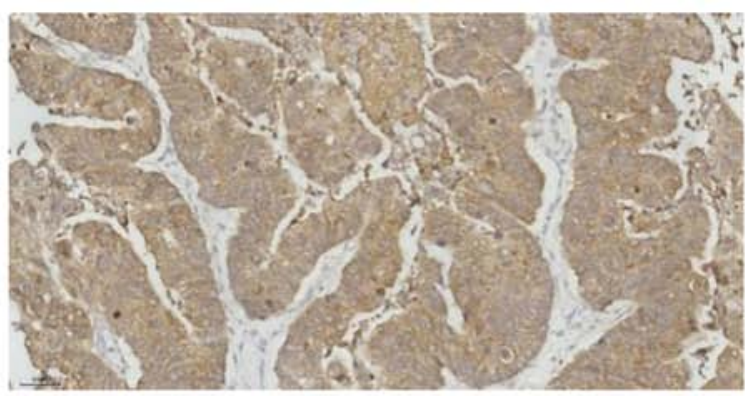

C

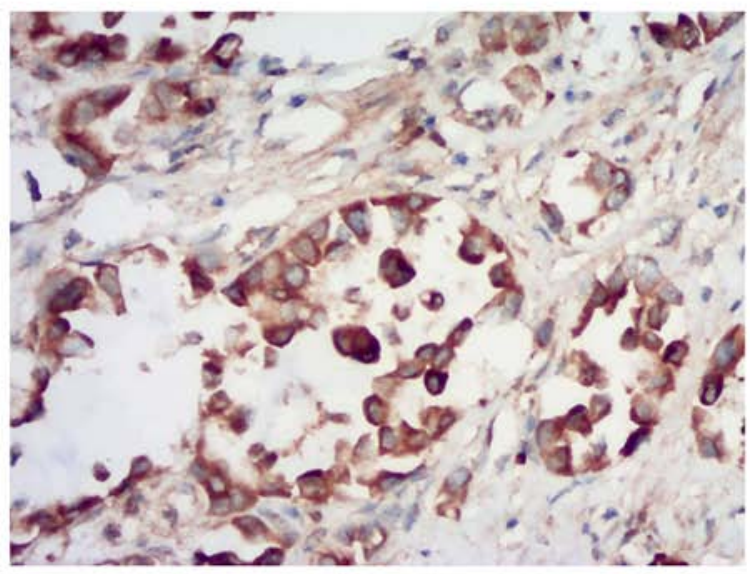

B

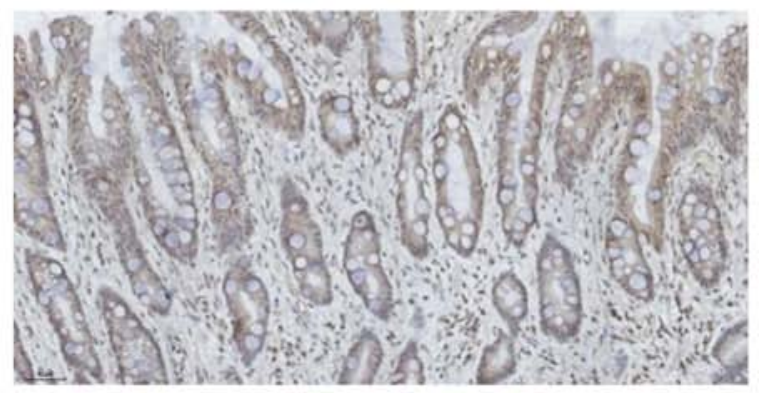

D

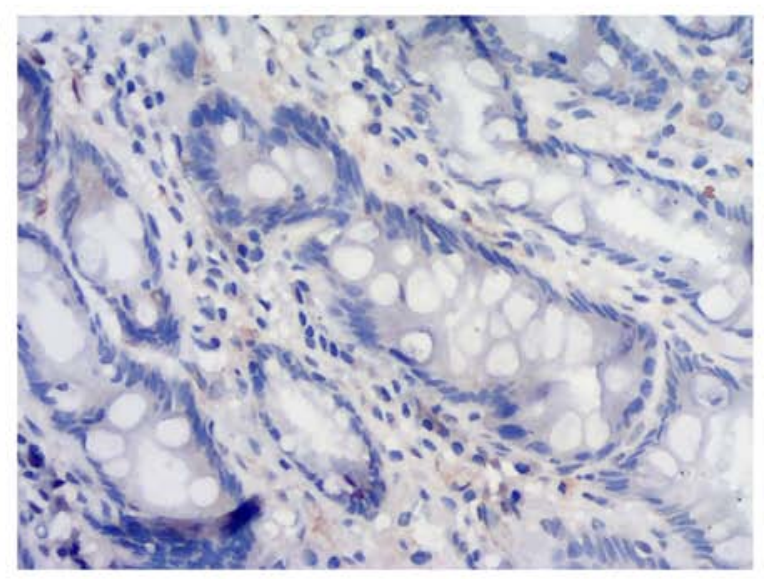

Figure 1. Representative images of ASAP1 and FAK protein expression in GC tissues. (A) Positive expression of ASAP1 in GC tissues. ASAP1 expression in GC tissues was positive, with a brown/yellow signal detected in the cytoplasm, as assessed by microscopy. (B) Negative expression of ASAP1 in paraneoplastic tissues. Cytoplasmic signal for ASAP1 was negative in normal tissues adjacent to the cancer. (C) Positive expression of FAK in GC tissues. FAK expression in GC tissues was positive, with a brown/yellow signal detected in the cytoplasm, as assessed by microscopy. (D) Negative expression of FAK in paraneoplastic tissues. Cytoplasmic staining of FAK was negative in normal tissues adjacent to the cancer. Magnification, x200. GC, gastric cancer; ASAP1, adenosine diphosphate ribosylation factor guanylate kinase 1; FAK, focal adhesion kinase.

\section{Results}

Protein expression of ASAPI and FAK in GC tissues. The expression levels of ASAP1 and FAK were detected in GC tissues, and a brown/yellow signal was identified in the cytoplasm of positive cells by microscopy. Most of the positive cells for ASAP1 (Fig. 1A) and FAK (Fig. 1C) were found in tumor tissues. however, a limited number of cells presenting a low signal for ASAP1 (Fig. 1B) and FAK (Fig. 1D) were found in normal tissues adjacent to cancerous tissues. The positive expression rates of ASAP1 in $32 \mathrm{GC}$ and normal gastric mucosal tissues were 59.4\% (19/32) and 28.1\% (9/32), respectively, and the difference was statistically significant $\left(\chi^{2}=6.349 ; \mathrm{P}=0.012\right)$. The positive expression rate of FAK was $68.8 \%(22 / 32)$ and $40.6 \%(13 / 32)$ in GC and normal gastric mucosal tissues, respectively, and the difference was statistically significant $\left(\chi^{2}=5.107 ; \mathrm{P}=0.024\right)$. As presented in Table II, the expression levels of ASAP1 and FAK in GC tissues were significantly associated with depth of invasion, lymph node metastasis, TNM stage and differentiation $(\mathrm{P}<0.05)$.

Association between ASAPI and FAK expression in GC tissues. Among the $19 \mathrm{GC}$ tissues with positive ASAP1 expression, 19 were positive for FAK (100.0\%). Among the 13 GC tissues with negative ASAP1 expression, 3 were positive for FAK $(23.1 \%) . \kappa$ analysis indicated that the expression levels of ASAP1 were associated with FAK expression in GC tissues $(\kappa=0.798 ; \mathrm{P}<0.001$; Table III).
Association between ASAP1 expression, FAK expression and $G C$ prognosis. The Kaplan-Meier survival curve analysis revealed that ASAP1 and FAK expression levels were negatively associated with 5-year DFS time in patients with GC. The 5-year DFS rate was $57.9 \%$ (11/19) in the ASAP1-positive group and $92.3 \%(12 / 13)$ in the negative group. The difference was statistically significant $\left(\log\right.$-rank $\chi^{2}=4.721 ; \mathrm{P}=0.030$; Fig. 2A). The 5-year DFS rate was $63.6 \%(14 / 22)$ in the FAK-positive group and $90.0 \%(9 / 10)$ in the negative group. The difference was not statistically significant (log-rank $\chi^{2}=2.472 ; \mathrm{P}=0.116$; Fig. $2 \mathrm{~B}$ ).

The 5-year OS rate of patients with positive and negative ASAP1 expression was 52.8\% (10/19) and 100.0\% (13/13), respectively, and the difference was statistically significant (log-rank $\chi^{2}=7.988 ; \mathrm{P}=0.005$; Fig. 2C). The 5-year OS rate of patients with positive and negative FAK expression was $59.1 \%$ $(13 / 22)$ and $100.0 \%(10 / 10)$, respectively, and the difference was statistically significant $\left(\log\right.$-rank $\chi^{2}=5.092 ; \mathrm{P}=0.024$; Fig. 2D).

\section{Discussion}

GC is one of the most common malignant tumors that poses a severe threat to human health. Genome-driven targeted cancer therapies may provide novel and promising strategies for cancer prevention and control (16). In 2009, the American Society of Clinical Oncology reported the first targeted therapy for GC in the 'trastuzumab for gastric cancer trial', demonstrating that trastuzumab combined with chemotherapy 
Table II. Association between ASAP1 and FAK expression and clinicopathological features of patients with gastric cancer ( $\mathrm{n}=32$ ).

\begin{tabular}{|c|c|c|c|c|c|c|}
\hline \multirow[b]{2}{*}{ Characteristic } & \multicolumn{3}{|c|}{ ASAP1 } & \multicolumn{3}{|c|}{ FAK } \\
\hline &,$- \mathrm{n}$ &,$+ \mathrm{n}$ & P-value &,$- \mathrm{n}$ &,$+ \mathrm{n}$ & P-value \\
\hline \multicolumn{7}{|l|}{ Age, years } \\
\hline$<60$ & 6 & 3 & 0.109 & 4 & 5 & 0.407 \\
\hline$\geq 60$ & 7 & 16 & & 6 & 17 & \\
\hline \multicolumn{7}{|l|}{ Sex } \\
\hline Female & 5 & 4 & 0.427 & 4 & 5 & 0.407 \\
\hline Male & 8 & 15 & & 6 & 17 & \\
\hline \multicolumn{7}{|l|}{ Invasion depth } \\
\hline $\mathrm{pT} 1+2$ & 11 & 3 & $<0.001$ & 9 & 5 & 0.001 \\
\hline pT3+4 & 2 & 16 & & 1 & 17 & \\
\hline \multicolumn{7}{|l|}{ Lymph node metastasis } \\
\hline No & 8 & 2 & 0.005 & 7 & 3 & 0.003 \\
\hline Yes & 5 & 17 & & 3 & 19 & \\
\hline \multicolumn{7}{|l|}{ TNM stage } \\
\hline $\mathrm{I}+\mathrm{II}$ & 11 & 4 & 0.001 & 9 & 6 & 0.002 \\
\hline III+IV & 2 & 15 & & 1 & 16 & \\
\hline \multicolumn{7}{|l|}{ Degree of differentiation } \\
\hline Moderate/high & 13 & 4 & $<0.001$ & 10 & 7 & $<0.001$ \\
\hline Undifferentiated/low & 0 & 15 & & 0 & 15 & \\
\hline
\end{tabular}

Data were analyzed by Fisher's exact probability test. pT1+2, tumor invades the submucosa or the muscularis propria; pT $3+4$, tumor invades the subserosa, non-peritonealised pericolic or perirectal tissues, or tumor directly invades other organs or structures and/or perforates the visceral peritoneum; -, negative; +, positive; ASAP1, adenosine diphosphate ribosylation factor guanylate kinase 1; FAK, focal adhesion kinase.

Table III. Association between ASAP1 and FAK expression in gastric cancer tissues.

\begin{tabular}{lrrrrr}
\hline & \multicolumn{2}{c}{ FAK expression } & & & \\
\cline { 2 - 3 } ASAP1 expression & Positive & Negative & Total & & P-value \\
\hline Positive & 19 & 0 & 19 & & \\
Negative & 3 & 10 & 13 & 0.798 & $<0.001$ \\
Total & 22 & 10 & 32 &
\end{tabular}

ASAP1, adenosine diphosphate ribosylation factor guanylate kinase 1; FAK, focal adhesion kinase.

as first-line treatment may improve the survival of human epidermal growth factor receptor 2 (HER2)-positive patients with advanced GC (17). However, the global positive rate of HER2 in advanced GC is only $25 \%$, and half of these patients exhibit a poor response to trastuzumab due to unknown causes (18). To the best of our knowledge, the only first-line targeted drug for GC is trastuzumab, the only second-line drug is ramucirumab and the only third-line drug is apatinib (19). Therefore, the available targeted treatments for GC are limited compared with those for other types of cancer, and further studies are required to develop novel therapeutic strategies to improve GC treatment.

ASAP1 is a phospholipid-dependent GTPase-activating protein (GAP) located on the long arm of chromosome
8, at 24.1-24.2. As a member of the ARF GAP family, ASAP1 hydrolyzes GTP to regulate actin re-organization and actin cytoskeletal dynamics, in this way controlling motility, regulating the formation of focal adhesions and invasive pseudopods, and contributing to the folding of the plasma membrane (20-24). In addition, ASAP1 binds to the $\mathrm{SH} 3$ domain-containing kinase-binding protein 1, the CD2-associated protein, cortactin, the CRK-like proto-oncogene adaptor protein and the SRC proto-oncogene non-receptor tyrosine kinase through its structural domains to exert its biological activities and to regulate cell invasion (25-27). Recent studies have described the association between ASAP1 and the biological features of malignant tumors. Müller et al (20) demonstrated that ASAP1 promotes 

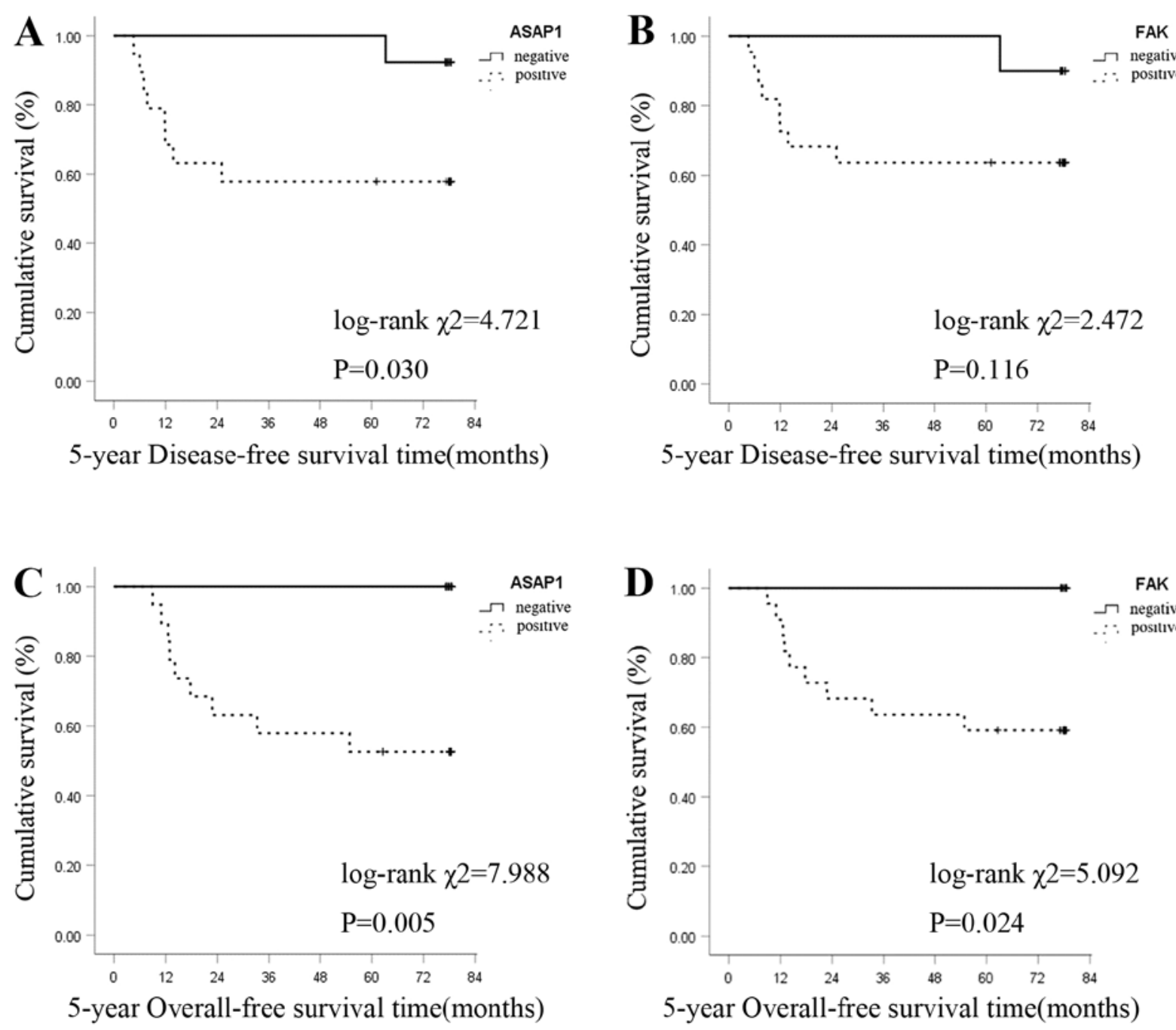

Figure 2. Survival curves of gastric carcinoma generated by the Kaplan-Meier method and the log-rank test. (A) Association between ASAP1 expression and 5 -year DFS time. The 5-year DFS rate was lower in the ASAP1-positive group compared with that in the ASAP1-negative group $\left(\log\right.$-rank $\left.\chi^{2}=4.721, \mathrm{P}=0.030\right)$. (B) Association between FAK expression and 5-year DFS time. The 5-year DFS rate was lower in the FAK-positive group than that in the FAK-negative group ( $\log$-rank $\chi^{2}=2.472, \mathrm{P}=0.116$ ). (C) Association between ASAP1 expression and 5-year OS time. The 5-year OS rate was lower in the ASAP1-positive group than that in ASAP1-negative group (log-rank $\chi^{2}=7.988, \mathrm{P}=0.005$ ). (D) Association between FAK expression and 5-year OS time. The 5-year OS rate was lower in the FAK-positive group than that in the FAK-negative group $\left(\log\right.$-rank $\left.\chi^{2}=5.092, \mathrm{P}=0.024\right)$. OS, overall survival; DFS, disease-free survival; ASAP1, adenosine diphosphate ribosylation factor guanylate kinase 1; FAK, focal adhesion kinase.

the invasion of colorectal cancer cells in vitro and stimulates the metastasis of colorectal cancer cells in vivo. Hou et al (5) revealed that ASAP1 expression in ovarian cancer tissues is significantly higher than that in normal ovarian tissues. In-depth analysis has demonstrated that positive ASAP1 expression is an indicator of poor prognosis in ovarian cancer and is an independent prognostic factor for the OS rate of patients with ovarian cancer. In addition, Liu et al (28) demonstrated that downregulation of ASAP1 expression by RNA interference inhibits cell proliferation and migration. However, to the best of our knowledge, no previous studies have investigated ASAP1 expression in GC tissues. In the present study, the expression levels of ASAP1 in GC tissues were significantly higher than those in tumor-adjacent normal gastric mucosal tissues $(\mathrm{P}=0.012)$. ASAP1 expression in GC tissues from patients with a T3+T4 infiltration depth was higher compared with that in patients with a T1+T2 infiltration depth $(\mathrm{P}<0.001)$. Furthermore, ASAP1 expression in GC tissues from patients with lymph node metastasis was higher than that of patients without lymph node metastasis $(\mathrm{P}=0.005)$. ASAP1 expression in $\mathrm{GC}$ tissues from patients at stages III and IV was significantly higher than that of patients at stages I and II $(\mathrm{P}=0.001)$. Compared with moderate or higher degree of differentiation, patients with undifferentiated or lower degree of differentiation had a higher ASAP1 expression levels $(\mathrm{P}<0.001)$. The DFS rates of patients with positive and negative ASAP1 expression were $57.9 \%(11 / 19)$ and $92.3 \%$ (12/13), respectively, with a significant difference between the two groups $(\mathrm{P}=0.030)$. The OS rates of patients with positive and negative ASAP1 expression were $52.8 \%$ $(10 / 19)$ and $100.0 \%(13 / 13)$, respectively, with a significant difference between the two groups $(\mathrm{P}=0.005)$. Therefore, the present results suggested that ASAP1 may serve important roles in the growth, invasion and metastasis of malignant gastric tumors, and may represent a novel molecular marker for evaluating the biological behavior and prognosis of GC. 
FAK was first identified and cloned by Schaller et al (29) from v-src-transfected chicken embryo fibroblasts in the 1990s. The human $F A K$ gene is located on the long arm of chromosome 8 (8q24). The FAK protein is a non-receptor tyrosine protein kinase with six tyrosine sites that can be phosphorylated: Tyr397 and Tyr407 are located at the amino terminus, Tyr576 and Tyr577 are located in the activation loop of the kinase domain, and Tyr861 and Tyr925 are located at the carboxyl terminus. Activated FAK participates in tumor proliferation, growth, invasion and metastasis via multiple signaling pathways, such as the FAK-Ras-PK, FAK-PI3K and FAK-STAT pathways (12). A study by Weiner et al (30) published in 1993 revealed that FAK expression is increased in invasive tumors and that positive FAK expression is present in all metastatic tumors, suggesting that it may promote tumor cell invasion and metastasis. A study by Miyazaki et al (31) published in 2003 demonstrated that FAK upregulation is associated with infiltration depth, lymph node metastasis and the number of metastatic lymph nodes in esophageal cancer. In 2010, Park et al (32) used immunohistochemistry to detect FAK expression in 444 surgically resected GC tissues. Additionally, a study by Lai et al (33) revealed that FAK can be autophosphorylated at the 397-tyrosine residue and that after this activation it initiates processes such as the proliferation, invasion and migration of GC cells. A study by Fan et al (11) in 2013 demonstrated that FAK activation increases endogenous A2 protein phosphorylation and leads to changes in epithelial-mesenchymal transition markers, including matrix metalloproteases, thereby inducing tumorigenesis and tumor progression. A study by Wang et al (34) in 2019 revealed that FAK binds directly to microRNA-1224 to inhibit the activation of the STAT3 and NF- $\kappa \mathrm{B}$ pathways, thereby suppressing the metastasis of GC. The present results suggested that the expression levels of FAK in GC tissues were significantly upregulated compared with those in tumor-adjacent normal gastric mucosal tissues $(\mathrm{P}=0.024)$. Additionally, FAK expression was associated with depth of infiltration, lymph node metastasis, increased tumor stage and decreased differentiation degree. The OS rates of patients with positive and negative FAK expression were $59.1 \%(13 / 22)$ and $100.0 \%(10 / 10)$, respectively, with a significant difference between the two groups $(\mathrm{P}=0.024)$. The present results suggested that FAK may serve a role in the tumorigenesis and progression of GC. Abnormal FAK expression may lead to more malignant GC cell proliferation, growth, invasion and metastasis. Therefore, FAK may be useful as a novel molecular marker to evaluate the biological behavior and prognosis of GC.

ASAP1 is a multi-domain protein with rich in proline structural regions which binds to various structural proteins to exert its biological function. ASAP1 interacts with non-muscle myosin II-A to regulate actin cytoskeleton remodeling and the transport of integrin, thereby affecting cell invasion and metastasis $(35,36)$. FAK, as a key molecule in integrin-dependent signal transduction pathways, serves an important role in the binding between integrin and ligands, thus promoting the formation of focal adhesions (37). A study by Liu et al (28) used yeast two-hybrid screening and a co-immunoprecipitation assay to demonstrate a direct interaction between ASAP1 and FAK. In the present study, 29 samples were found to co-express ASAP1 and FAK in GC tissues, which resulted in a $\kappa$ value of 0.798 $(\mathrm{P}<0.001)$, indicating that ASAP1 expression was associated with FAK expression and that ASAP1 and FAK promoted the pathophysiology of malignant GC. The present results suggested an association between ASAP1 and FAK, which affected the pathophysiology of malignant GC. Therefore, ASAP1 may promote the malignant phenotype of GC cells through FAK.

In conclusion, ASAP1 and FAK were highly expressed in GC tissues and were associated with degree of invasion, lymph node metastasis, pathological staging and degree of differentiation in the present study. In addition, positive expression of ASAP1 and FAK may be risk factors for the prognosis of patients with GC. The present findings suggested that ASAP1 and FAK may synergistically promote the tumorigenesis, tumor progression, invasion and metastasis of GC, and are closely associated with the survival of patients with GC. Although the sample size was too small, which was one of the limitations of the present study, it provided a basis for a follow-up study on the association between ASAP1, FAK and GC. ASAP1 and FAK may represent novel molecular markers for the pathophysiology and prognosis of GC. In addition, further in vitro and in vivo studies investigating the molecular mechanisms of ASAP1 and FAK in the tumorigenesis and tumor progression of GC may help to elucidate the pathophysiology of malignant GC. Furthermore, additional studies may provide important information for the early diagnosis, treatment and prognostic assessment of GC, as well as novel molecular targets and therapeutic strategies to develop novel drugs to treat GC.

\section{Acknowledgements}

Not applicable.

\section{Funding}

The present study was partially supported by the Health-Education Joint Research Project of Fujian Province (grant no. WKJ2016-2-23), the Science Technology Innovation Joint Project Foundation of Fujian Province (grant no. 2017Y9003), the Startup Fund for Scientific Research, Fujian Medical University (grant no. 2017XQ2037), the Science Technology Innovation Joint Project Foundation of Fujian Province (grant no. 2018Y9038) and the Program for Innovative Research Team in Science and Technology in Fujian Province University.

\section{Availability of data and materials}

The datasets used and/or analyzed during the present study are available from the corresponding author on reasonable request.

\section{Authors' contributions}

SY and XC conceived and supervised the project. QL, SZ, DZ and FY performed the experiments and analyzed the data. QL, SZ and DZ wrote the manuscript. All authors read and approved the final manuscript.

\section{Ethics approval and consent to participate}

Ethical approval for the present study was obtained from the Union Hospital of Fujian Medical University Ethics 
Committee (Fuzhou, China). All patients or their guardians provided written informed consent.

\section{Patient consent for publication}

Not applicable.

\section{Competing interests}

The authors declare that they have no competing interests.

\section{References}

1. Ferlay J, Soerjomataram I, Dikshit R, Eser S, Mathers C, Rebelo M, Parkin DM, Forman D and Bray F: Cancer incidence and mortality worldwide: Sources, methods and major patterns in GLOBOCAN 2012. Int J Cancer 136: E359-E386, 2015.

2. Li J, Meng Q, Sun Y and Qing H: Inhibition of focal adhesion kinase induces apoptosis in human gastric carcinoma cells (SGC-7901). Mol Biol Rep 40: 401-406, 2013.

3. Li Z, Lei H, Luo M, Wang Y, Dong L, Ma Y, Liu C, Song W, Wang F, Zhang J, et al: DNA methylation downregulated mir-10b acts as a tumor suppressor in gastric cancer. Gastric Cancer 18: 43-54, 2015.

4. Kanda M and Kodera Y: Recent advances in the molecular diagnostics of gastric cancer. World J Gastroenterol 21: 9838, 2015.

5. Hou T, Yang C, Tong C, Zhang H, Xiao J and Li J: Overexpression of ASAP1 is associated with poor prognosis in epithelial ovarian cancer. Int J Clin Exp Pathol 7: 280, 2014.

6. Zhang L, Shi SB, Zhu Y, Qian TT and Wang HL: Long non-coding RNA ASAP1-IT1 promotes cell proliferation, invasion and metastasis through the PTEN/AKT signaling axis in non-small cell lung cancer. Eur Rev Med Pharmacol Sci 22: 142-149, 2018

7. Randazzo PA, Inoue $\mathrm{H}$ and Bharti S: Arf GAPs as regulators of the actin cytoskeleton. Biol Cell 99: 583-600, 2007.

8. Zhang T, Zhao G, Yang C, Dong P, Watari H, Zeng L, Pfeffer LM and Yue J: Lentiviral vector mediated-ASAP1 expression promotes epithelial to mesenchymal transition in ovarian cancer cells. Oncol Lett 15: 4432-4438, 2018.

9. Yang L, Xue Y, Liu J, Zhuang J, Shen L, Shen B, Yan J and Guo H: Long noncoding RNA ASAP1-IT1 promotes cancer stemness and predicts a poor prognosis in patients with bladder cancer. Neoplasma 64: 847-855, 2017.

10. Sulzmaier FJ, Jean C and Schlaepfer DD: FAK in cancer: Mechanistic findings and clinical applications. Nat Rev Cancer 14: 598, 2014.

11. Fan H, Zhao X, Sun S, Luo M and Guan JL: Function of focal adhesion kinase scaffolding to mediate endophilin A2 phosphorylation promotes epithelial-mesenchymal transition and mammary cancer stem cell activities in vivo. J Biol Chem 288: 3322-3333, 2013.

12. Fu W, Hall JE and Schaller MD: Focal adhesion kinase-regulated signaling events in human cancer. Biomol Concepts 3: 225-240, 2012.

13. Thanapprapasr D, Previs RA, Hu W, Ivan C, Armaiz-Pena GN, Dorniak PL, Hansen JM, Rupaimoole R, Huang J, Dalton HJ, et al: PTEN expression as a predictor of response to focal adhesion kinase inhibition in uterine cancer. Mol Cancer Ther 14: 1466-1475, 2015.

14. Zeng XQ, Li N, Ma LL, Tseng YJ, Zhao NQ and Chen SY: Prognostic value of focal adhesion kinase (FAK) in human solid carcinomas: A meta-analysis. PLoS One 11: e0162666, 2016.

15. Sobin L: International Union Against Cancer (UICC) TNM classification of malignant tumours. Oesophagus including Oesophagogastric Junction: 66-72, 2009.

16. Buqué A, Bloy N, Aranda F, Castoldi F, Eggermont A, Cremer I, Fridman WH, Fucikova J, Galon J, Marabelle A, et al: Trial watch: Immunomodulatory monoclonal antibodies for oncological indications. Oncoimmunology 4: e1008814, 2015.

17. Petrelli NJ, Winer EP, Brahmer J, Dubey S, Smith S, Thomas C, Vahdat LT, Obel J, Vogelzang N, Markman M, et al: Clinical Cancer Advances 2009: Major research advances in cancer treatment, prevention, and screening-a report from the American Society of Clinical Oncology. J Clin Oncol 27: 6052-6069, 2009.
18. Yazici O, Sendur MA, Ozdemir N and Aksoy S: Targeted therapies in gastric cancer and future perspectives. World J Gastroenterol 22: 471-489, 2016.

19. Feng R, Zhang X and Yang S: Research status quo and progression in targeted therapy for advanced gastric cancer. Zhonghua Wei Chang Wai Ke Za Zhi 19: 1191-1196, 2016 (In Chinese).

20. Müller T, Stein U, Poletti A, Garzia L, Rothley M, Plaumann D, Thiele W, Bauer M, Galasso A, Schlag P, et al: ASAP1 promotes tumor cell motility and invasiveness, stimulates metastasis formation in vivo, and correlates with poor survival in colorectal cancer patients. Oncogene 29: 2393-2403, 2010.

21. Randazzo PA, Andrade J, Miura K, Brown MT, Long YQ, Stauffer S, Roller P and Cooper JA: The Arf GTPase-activating protein ASAP1 regulates the actin cytoskeleton. Proc Natl Acad Sci USA 97: 4011-4016, 2000.

22. Liu Y, Yerushalmi GM, Grigera PR and Parsons JT: Mislocalization or reduced expression of Arf GTPase-activating protein ASAP1 inhibits cell spreading and migration by influencing Arf1 GTPase cycling. J Biol Chem 280: 8884-8892, 2005.

23. Inoue $\mathrm{H}$ and Randazzo PA: Arf GAPs and their interacting proteins. Traffic 8: 1465-1475, 2007.

24. Nie Z and Randazzo PA: Arf GAPs and membrane traffic. J Cell Sci 119: 1203-1211, 2006.

25. Brown MT, Andrade J, Radhakrishna H, Donaldson JG, Cooper JA and Randazzo PA: ASAP1, a phospholipid-dependent arf GTPase-activating protein that associates with and is phosphorylated by Src. Mol Cell Biol 18: 7038-7051, 1998.

26. Kowanetz K, Husnjak K, Höller D, Kowanetz M, Soubeyran P, Hirsch D, Schmidt MHH, Pavelic K, De Camilli P, Randazzo PA and Dikic I: CIN85 associates with multiple effectors controlling intracellular trafficking of epidermal growth factor receptors. Mol Biol Cell 15: 3155-3166, 2004.

27. Oda A, Wada I, Miura K, Okawa K, Kadoya T, Kato T, Nishihara H, Maeda M, Tanaka S, Nagashima K, et al: CrkL directs ASAP1 to peripheral focal adhesions. J Biol Chem 278: 6456-6460, 2003.

28. Liu Y, Loijens JC, Martin KH, Karginov AV and Parsons JT: The association of ASAP1, an ADP ribosylation factor-GTPase activating protein, with focal adhesion kinase contributes to the process of focal adhesion assembly. Mol Biol Cell 13: 2147-2156, 2002.

29. Schaller MD, Hildebrand JD and Parsons JT: Complex formation with focal adhesion kinase: A mechanism to regulate activity and subcellular localization of Src kinases. Mol Biol Cell 10: 3489-3505, 1999.

30. Weiner TM, Craven RJ, Craven RJ and Cance WG: Expression of focal adhesion kinase gene and invasive cancer. Lancet 342: 1024-1025, 1993

31. Miyazaki T, Kato H, Nakajima M, Sohda M, Fukai Y, Masuda N, Manda R, Fukuchi M, Tsukada K and Kuwano H: FAK overexpression is correlated with tumour invasiveness and lymph node metastasis in oesophageal squamous cell carcinoma. $\mathrm{Br}$ J Cancer 89: 140, 2003.

32. Park JH, Lee BL, Yoon J, Kim J, Kim MA, Yang HK and Kim WH: Focal adhesion kinase (FAK) gene amplification and its clinical implications in gastric cancer. Hum Pathol 41: 1664-1673, 2010.

33. Lai HC, Zhuang LF, Liu X, Wieland M, Zhang ZY and Zhang ZY: The influence of surface energy on early adherent events of osteoblast on titanium substrates. J Biomed Mater Res A 93: 289-296, 2010.

34. Wang J, Wen T, Li Z, Che X, Gong L, Yang X, Zhang J, Tang H, He L, Qu X and Liu Y: MicroRNA-1224 inhibits tumor metastasis in intestinal-type gastric cancer by directly targeting FAK. Front Oncol 9: 222, 2019.

35. Yoon HY, Jacques K, Nealon B, Stauffer S, Premont RT and Randazzo P: Differences between AGAP1, ASAP1 and Arf GAP1 in substrate recognition: Interaction with the N-terminus of Arf1. Cell Signal 16: 1033-1044, 2004.

36. Vitali T, Girald-Berlingeri S, Randazzo PA and Chen PW: Arf GAPs: A family of proteins with disparate functions that converge on a common structure, the integrin adhesion complex. Small GTPases 10: 280-288, 2019.

37. Brami-Cherrier K, Gervasi N, Arsenieva D, Walkiewicz K, Boutterin MC, Ortega A, Leonard PG, Seantier B, Gasmi L, Bouceba T, et al: FAK dimerization controls its kinase-dependent functions at focal adhesions. EMBO J 33: 356-370, 2014. 